\title{
Germanica
}

philosophique

\section{Wir Überlebenden: Zur Heiterkeit des Unmöglichen}

We Survivors: On the Cheerfulness of the Impossible

Nous, les survivants : De l'allégresse de l'impossible

\section{Jana V. Schmidt}

\section{OpenEdition \\ Journals}

Édition électronique

URL : http://journals.openedition.org/germanica/5435

DOI : 10.4000/germanica.5435

ISSN : 2107-0784

\section{Éditeur}

Université de Lille

\section{Édition imprimée}

Date de publication : 31 décembre 2018

Pagination : 147-162

ISBN : 978-2-913857-42-1

ISSN : 0984-2632

\section{Référence électronique}

Jana V. Schmidt, „Wir Überlebenden: Zur Heiterkeit des Unmöglichen“, Germanica [Online], 63 | 2018,

Online erschienen am: 01 Januar 2021, abgerufen am 22 Januar 2021. URL: http://

journals.openedition.org/germanica/5435; DOl: https://doi.org/10.4000/germanica.5435

\section{(c) Tous droits réservés}




\title{
Wir Überlebenden: Zur Heiterkeit des Unmöglichen
}

\author{
Jana V. SCHMIDT \\ California State University, Los Angeles
}

\section{„Seltsame wirkliche Namen von Menschen“}

Im Archiv des 1988 in London verstorbenen tschechischen Schriftstellers H.G. Adler befindet sich eine zerschlissene Mappe, die den Titel „Seltsame wirkliche Namen von Menschen“ trägt ${ }^{1}$. Die hier gesammelten ulkigen Namen von österreichischen Musikpädagogen, britischen Fleischereimeistern und einstigen deutschen SS-Hauptscharführern mögen Adler als Inspiration für seine Romane und Kurzgeschichten gedient haben. Hat man sich schmunzelnd von Egon Dahinten bis Elfriede Vollenhals gelesen, die Todesanzeigen einer Riesenradbesitzersgattin und der ,Wohlgeborenen Frau Maria Anna von Sausenhoven auf Sausenhofen" bestaunt, stößt der Leser am Boden der Mappe auf einige eng getippte Namenslisten auf dünnem Fliegenpapier. In alphabetischer, nach Nachnamen sortierter Reihenfolge stehen hier die Namen von knapp vierhundert Personen in zwei Spalten auf vier Seiten, von Sarina Aberbauch bis Jonas Zimmerspitz. Es ist ein bunter Baukasten von poetischen, lustigen, manchmal vulgären und seltenen Wortschöpfungen, welcher die strenge Ordnung der Zeilen Lügen zu

1. - Deutsches Literaturarchiv Marbach, Adler AII 15. Dem Marbacher Archiv gebührt mein herzlicher Dank für allerlei Hilfeleistungen bei der Recherche und einen idealen Arbeitsplatz während meines Aufenthalts als Marbach-Stipendiatin. 
strafen scheint. Namen wie Augapfel, Feuereisen, Balsambaum und Bogenglück klingen romantisch, solche wie Dreinudel, Affenkraut und Bauchwitz amüsant, während andere Namen einem bürokratischen Geist entsprungen zu sein scheinen: Durchschlag, Beamt, Dintenfass und Dienstfertig. Sie zeugen auf besonders eindrückliche Weise von der Geschichte jüdischer Namensgebung in Mittel- und Osteuropa, von den Emanzipationsgesetzen, die den Besitz eines Nachnamens, oft in Verbindung mit Staatsbürgerschaft, auch für die jüdische Minderheit in Galizien, der Bukowina, Polen, Preußen und Russland zum Mandat machten. Die „Taufe“ durch Standesbeamte, deren Fantasie nur durch Bestechungsgelder zu besänftigen war, markierte die Träger der neuen Namen und deren Kinder und Kindeskinder deutlich ${ }^{2}$. Jüdische Nachnamen reflektieren das Beziehungsgeflecht von souveräner Macht, Gemeinschaft und Ausschluss in der Geschichte der europäischen Nationalstaaten.

Zugleich Teil und Unterbrechung dieser Geschichte zeichnet Adlers Assemblage eine ganz besondere Gruppe aus: Diese jüdischen Namen gehören, einer handschriftlich hinzugefügten Überschrift nach, den Überlebenden des Konzentrationslagers Theresienstadt, in dem Adler zweieinhalb Jahre gefangen war und von dem aus er 1944 weiter, erst nach Auschwitz, dann in ein Nebenlager von Buchenwald (Niederorschel) und letztlich in das Arbeitslager Langenstein verschleppt wurde. Dort begann er, Gedichte, Kurzgeschichten und den Entwurf eines Romans (der unveröffentlichte Raoul Feuerstein, der von der quälenden Besessenheit des Protagonisten durch seinen eigenen Namen handelt) zu schreiben. Unter unvorstellbaren Bedingungen tippte er als „Hallenschreiber“ bei den Junkers-Flugzeugwerken Gedichte wie Inventarlisten und verdiente sich als „Hofdichter“ eines Lagerkapos die Extrarationen, die ihm und seinem Gehilfen, dem späteren serbischen Schriftsteller Ivan Ivanji, das Leben retteten ${ }^{3}$. Neben den in Langenstein entstandenen Texten gelang es Adler auch, seine Manuskripte aus Theresienstadt, die er vor seinem Abtransport dem Rabbiner, ehemaligem Präsidenten der Reichsvertretung der Juden und Mitglied des Ältestenrats im KZ Theresienstadt, Leo Baeck, anvertraut hatte, zu retten. Im Sommer 1945 besuchte er zu diesem Zweck das ehemalige Konzentrationslager, in dem die sogenannte Evidenzabteilung (die kurz zuvor noch die Deportationslisten zusammengestellt hatte), sowie Reste der komplizierten „Selbstverwaltung“ des Lagers auch nach der Übernahme durch

2. - Benzion C. Kaganoff, „Jewish Surnames through the Ages“, Commentary 22:3 (Sep. 1, 1956), S. 249-259.

3. - Ivanji zitiert in Jürgen Serke, ,H.G. Adler. Der versteinerte Jüngling, der ein weiser Mann wurde“, in: Ders., Böhmische Dörfer. Wanderungen durch eine verlassene literarische Landschaft, Wien und Hamburg, Paul Szolnay, 1987, S. 326-343, hier S. 336. 
das Internationale Rote Kreuz und die sowjetische Armee noch weiter arbeiteten. Als Mitarbeiter im Prager Jüdischen Museum kurz nach Kriegsende gründete Adler das Archiv der Theresienstädter Dokumente, auf dem seine wichtige Studie Theresienstadt 1941-1945. Das Antlitz einer Zwangsgemeinschaft beruht und durch die Adler womöglich an die Namenslisten der überlebenden Lagerinsassen gelangte.

Wovon zeugen aber diese „seltsamen“ Namen? Welche Eigenschaft des Namens oder der Gemeinschaft, die Namen stiften, erlaubt es Adler, dem späteren Historiker der NS-Verwaltung, aus hunderten von Listen, Karteikarten und dem Katalog der Verfolgung, Privilegierung und des Mords nur die kuriosesten Nachnamen der Überlebenden herauszupicken? Wie verhält sich Adlers Sammlung, und die heiter-ironische Geste der Aufstellung einer Überlebendenliste, gegenüber der identifizierenden (sprich tödlichen) Funktion des Namens in den Lagern einerseits? Und in welchem Verhältnis steht sie andererseits zu dem Umstand, dass der Name sich gewöhnlich genau ,gegen den Hintergrund einer originären Uneigentlichkeit oder Metaphorizität" ${ }^{4}$ abhebt? Als einziges Wort, das nichts bedeuten soll, ist der Name ein Platzhalter für ein stets abwesendes Subjekt - eine Logik, der sich Adlers Namen zu widersetzen scheinen. Ironisch weisen sie auf ein heiter-poetisches Hier: das Hier der sinnlich erfahrbaren Bildlichkeit des Signifikanten und das Hier der trotz alledem Überlebenden.

Die Liste steht jedoch nicht allein in einem Werk, das voll von heiteren Namenschöpfungen und ironischen Verfremdungen kollektiver Existenz ist. H.G. Adler stellt sich in seinen Romanen und kürzeren Texten der Frage, wie ,in Ermangelung eines pluralen Subjekts“ ${ }^{\circ}$ der Erfahrung über die Lager geschrieben werden kann. Er tut dies über seine besondere Innovation einer Erzählstimme, die sich an die Sprache der Vernichtung angleicht. Heiterkeit, so erweist sich, ist bei Adler der Versuch, das Verhältnis von menschlicher Bezüglichkeit und Ästhetik neu zu fassen.

\section{Die bittere Stimme}

Sprechende Namen finden sich in nahezu allen veröffentlichten Texten H.G. Adlers. So zum Beispiel in dem 1962 erschienen Roman Eine Reise, in dem eine Familie Lustig die "Reise' nach Ruhenthal

4. - So fasst Geoffrey Bennington einen Grundgedanken der Dekonstruktion zusammen: ,the proper name and proper meaning are only distinguished in secondary fashion against a background of originary impropriety or metaphoricity“. Vgl. Geoffrey Bennington, Jacques Derrida, Chicago, University of Chicago Press, 1993, S. 107.

5. - Jean-François Lyotard, „Streitgespräche, oder: Sätze bilden ,nach Auschwitz““, in: Elisabeth Weber, Georg Christoph Tholen (Hrsg.), Das Vergessene. Anamnesen des Undarstellbaren, Wien, Turia und Kant, 1997, hier S. 24. 
antritt, von der ausschließlich ihr ältester Sohn Paul zurückkehren wird. Indem Adler, wie Ruth Vogel-Klein zeigt, Eigennamen und Ortsnamen bewusst ,antiphrastisch“ einsetzt, erhöht er den verfremdenden Effekt der „Ballade“6, welcher durch zahlreiche erzählerische Nebenstränge, eine nicht-chronologische Struktur und eine zur Abstraktion neigende Sprache „ein[e] fremde Welt, die keiner historischen oder geographischen Realität mehr entspricht und nur in der Sprache Adlers ihren Bestand hat", konstruiert ${ }^{7}$. Die ironische Distanzierung über die Namenswahl führe ,durch die Diskrepanz zwischen dem Signifikant[en] und dem Signifikat einen Störfaktor im Text" ${ }^{\text {"8 }}$ ein, der zum Beispiel die vermeintlich neutrale Sprache der nationalsozialistischen Bürokratie entlarve. „Kontrastiv lässt Adler auch - in einer Sprachkritik der Behörden- und Verordnungssprache der Nazis - Form und Inhalt der Aussagen aufeinanderprallen. Adler entlarvt Verwaltung als Mordinstrument, indem er die unterschwellige Brutalität einer sich neutral gebenden Sprache anprangert"9.

So nimmt Eine Reise Verbote und Ordnung wörtlich, zeigt wie diese, hier in einer kleinen Satire auf die heilige Verkündung, den Betroffenen quasi als Wort Gottes erscheinen: „Sie [die ,Helden'] kamen meist am späten Abend oder auch während der Nacht, wenn sie ihre Botschaft brachten, der sich ein entsetztes Licht nicht versagte. "Du sollst nicht wohnen!'“ Doch über die Entlarvung der Täterlogik hinaus erfasst Eine Reise besonders die problematische Seite der Reaktion der Lustigs auf das ihnen auferlegte Verbot ihrer selbst. Auf die Nachricht, dass man die Wohnung verlassen, alle Habseligkeiten aufgeben und ,umgesiedelt“ werden soll, scheint die Familie völlig passiv zu reagieren: „Nichts ist unverständlich, denn alles wurde von den Stellen so gut bedacht, daß keine Schwierigkeiten entstehen. Wer die langen Listen durchblättert, findet alle Richtlinien. Du mußt dich nur entschließen und du bist frei, wenn du willig dem Zwange gehorchst" 10 . Aus wessen Sicht hier allerdings berichtet wird, ist über weite Strecken völlig unklar. Es ist die anonyme Stimme des ,kollektiven Unbewussten', die hier, von passiven

6. - Adler selbst lehnte die Bezeichnungen Roman und Erzählung ab und hätte Eine Reise als „Die Reise - Eine Ballade“ veröffentlicht. Dazu kam es aus verlegerischen bzw. rechtlichen Gründen aber nicht. Siehe Jeremy Adler, „Nur wer die Reise wagt, findet nach Hause“, in: H.G. Adler, Eine Reise, Wien, Paul Szolnay, 1999, S. 310.

7. - Ruth Vogel-Klein, „Eine fremde Welt: H.G. Adler, Eine Reise“, Germanica 42/2008, S. 6.

8. - Ruth Vogel-Klein, ,,Keine Anklage“? Der Deportationsroman Eine Reise (1951/1962) von H.G. Adler. Publikation und Rezeption“, in: Dies. (Hrsg.), Die ersten Stimmen. Deutschsprachige Texte zur Shoah 1945-1963, Würzburg, Königshausen und Neumann, 2010, S. 79-111.

9. - Ebd., S. 83.

10. - H.G. Adler, Eine Reise, a.a.O., S. 13. 
Satzkonstruktionen, dem Imperativ und einer Art gezielten Vermeidung oder Unterordnung des grammatischen Subjekts bestärkt, vor sich hin raunt. Sätze wie „Man hat euch nicht zu dieser Ernte bestellt"11, „Niemand erhört euch“, „Abfall seid ihr“12 dominieren den Ton der Narrative in blockartigen Einschüben. Selbst dort, wo ein „Wir“Erzähler sich scheinbar zu erkennen gibt, bleibt dieses Wir eigenartig körperlos:

Wir alle sind verboten, weil wir nicht die sind, die wir sein möchten, doch auch die möchten wir nicht sein, weil man uns zur Wunschlosigkeit angehalten hat. So sind wir nichts mehr als das Gehör, das zum Werkzeug des Gehorsams wird, erwartungsvolle Reisegefährten, deren Müdigkeit hinreichen würde, die gesamte Menschheit in Schlaf zu versetzen ${ }^{13}$.

Die Erzählstimme vollzieht auf der Ebene der Handlung die zunehmende Entrechtung der Lustigs nach, denn bald ist alles verboten: „Es wurden die Wege verboten“, aber auch Tag und Nacht, Büchereien, Wäschereien, Musik und Baden, Geld und Verkaufen ${ }^{14}$. An der Sammelstelle im technischen Museum angelangt, sind die Familienmitglieder bereits zu ,ehemalige[n] Menschen“ 15 mutiert.

Indem die Lustigs ohne Sympathie aus den Augen der Täter beschrieben und belächelt werden, zieht der Erzähler Parallelen zwischen dem Familienkollektiv und den ordnungsliebenden „Helden“ mit ihren Listen. Perspektivisch schwankt der Roman zwischen auktorialer und personaler Erzählstimme, zwischen der beschränkten Sicht eines Familienmitglieds und dem bedrohlichen Imperativ einer unidentifizierbaren Stimme: „Bedenke es wohl, welches Anrecht du hast, einen Aufenthalt zu genießen, wo du sein dürftest, bloß weil du sein darfst"16. Hier spricht, so scheint es, die Logik der Vernichtung. Dazwischen aber schiebt sich immer wieder eine Art Komplementärstück oder Echo im Chor der tödlichen Logik, das die zwanghafte Rechtfertigung der Gewalt durch die Opfer selbst bloßlegt.

In der Tat lässt sich das Wir der Opfer in Eine Reise kaum vom Wir der Täter unterscheiden. Die Zusammenstellung der Transportlisten durch die Judenräte im Roman reflektiert diese brutale Verflechtung in der ,Grauzone“: „Eines Tages hieß es, zweitausend Menschen müssen fort, kräftige, arbeitsfähige Männer und Frauen. Sucht sie euch selber

\footnotetext{
11. - Ebd., S. 57.

12. - Ebd., S. 91.

13. - Ebd., S 15.

14. - Ebd., S. 27.

15. - Ebd., S. 30.

16. - Ebd., S. 10.
} 
aus, ihr seid ja vierzigtausend Leute! [...] Fragt nicht nach den Gründen, fragt auch nicht wohin, genug: wir brauchen sie!"17 Als „Vormünder“ und strenge, aber gerechte Herren treten die „Helden“ auf, um den „Geistern“ angedeihen zu lassen, was nötig und gut für sie ist. Folglich ist die brave „Selbstbezwingung“ der Opfer gleichbedeutend mit deren Selbstverleugnung:

Wir lehren euch, was das Gesetz ist. Es ist Zwang. Es tritt stets ein, wenn er nicht gewollt ist, das ist sein Wesen, denn sonst gäbe es keinen Zwang auf Erden, und die Menschen könnten sich zu wohl fühlen. Sucht euch schnell aus, wen ihr zum Zwang verurteilen wollt! Das ist die Selbstbezwingung, in der die Starken siegen und die Schwachen fallen. Ihr bereitet vor! Die Reise machen wir euch dann ${ }^{18}$.

Adlers entscheidende Innovation - und Provokation! - in Eine Reise besteht in einer Erzählstimme, die Täter, Zuschauer und Opfer in einer Art Psychofusion zusammenführt. Noch gegen Ende des Romans, als nur ein einziges Mitglied der Familie, der erwachsene Sohn Paul, übriggeblieben ist, dringt die Stimme der Vernichtung in die Erzählung und in sein Bewusstsein. „Wen beklagst du denn? Nur Toren jammern, weil sie die Gewohnheit nicht entbehren können. Doch du hast keine Eigenschaften mehr, das macht dich lieb und wert [...] Uns kannst du vertrauen, es wird zu deinem Heile sein"19, flüstert die Stimme, die nun gar keine Gruppe mehr zu repräsentieren scheint, sondern ein Destillat des ,Rationalismus“ der Shoah darstellt, welches die Musealisierung des Massenmords antizipiert. „Nun siehst du, jetzt bist du vernünftig, du kleiner Toter, den wir wiegen. Wir machen mit dir, was wir begehren, und zaubern noch, dass es dein eigener Wunsch wird“20.

\section{„Der Älteste“}

H. G. Adlers Romane und Kurzgeschichten handeln von der Unhaltbarkeit eines kollektiven Subjekts in den Lagern und versuchen, dieser auch sprachlich Form zu geben. In der frühen, noch im Konzentrationslager Theresienstadt geschriebenen und erstmals 1964 in Der Fürst des Segens veröffentlichten Erzählung „Der Älteste“ etwa versammeln sich die Bewohner einer kleinen Gemeinschaft, um dem Vortrag ihres Anführers, dem „Ältesten“, zu lauschen. Der Greis soll, umringt von seinem „Hofstaat" und den gleichsam in Trance versetzten

17. - Ebd., S. 156.

18. - Ebd.

19. - Ebd., S. 243.

20. - Ebd., 244. David Rousset bezeichnet dieses Phänomen der Einwilligung als einen Effekt des totalitären Terrors, der in der systematischen Identitätszerstörung bestehe. Siehe Le jours de notre mort, Paris, Éditions du Pavois, 1947. 
Hörern, die Gebote der Gemeinschaft vortragen. Mechanisch-träge folgen die Untertanen dem Befehl; nur mehr "Gestalten“ haben sie aufgehört, sich eigene Urteile zu bilden: „Da diese Gestalten weder ihren Körper auflösen konnten noch einem schattenhaften Dasein sich durch Selbstmord entzogen, verharrten sie dumpf brütend und werkelten nach dem Klopfen eines abschnurrenden Uhrwerkes fort, das sie zwar nicht als Automaten, aber doch fast unbelebt und gemütlos erscheinen ließ“"21. Theatralisch spitzt sich die Erzählung auf den Auftritt des Alten zu: der pathetische, fast märchenhafte Ton der Geschichte scheint sich selbst zu ernst zu nehmen; die ehrfürchtige Andacht der Wartenden und die Würde des popenhaften Alten muten lachhaft an. Und dann ist es so weit: „In nomine Domini. Es muss jejrüßt werden. Wer auch vorbeikommt, langsam - schnell, leise - laut, hoch - jering, jroß - klein: jejrüßtmuß er werden. Jejrüßt, jejrüßt, jejrüßt!“ So und weiter - ,,mindestens eine Viertelstunde“ - in breitestem Berliner Latein. Der antiklimaktische Moment der Verkündigung entblößt die gesamte Ordnung der Ghetto-Bewohner als sinnfreie Satire auf ein tragisches Verständnis der Konzentrationslager. Dieser satirischen Haltung entsprechend nimmt die erzählerische Perspektive von „Der Älteste“ zunächst explizit Abstand von der Handlung. Die Rede ist von den kümmerlichen Mitgliedern der Zwangsgemeinschaft in der dritten Person: sie waren „dumpf brütend“, „fast unbelebt und gemütlos“, „gezwungen“ und „verstockt“. Adlers Geschichte verfährt mit den fiktionalen Bewohnern des Ghettos so unerbittlich wie es nur ein Überlebender der Konzentrationslager kann. Die Erfahrung des Massenmords, der Deportation und der erzwungenen Kooperation durch die Judenräte kann, das bestätigt Adlers Erzählung, niemals dazu dienen, Identifikation neu zu stiften. Sie kann für den Einzelnen nur zur angewiderten Abkehr von Gemeinschaft führen oder zur selbstzerstörerischen Scham vor seiner unvermeidbaren Komplizenschaft.

Und doch kann der Erzähler der Geschichte selbst seine Verachtung für die Zuhörer letztlich nicht aufrechterhalten. In einem in dieser Form unveröffentlichten Entwurf der Erzählung vom November 1942 (mit dem Vermerk „Umarbeitung Juli 43“), der sich unter den Papieren des Nachlasses von H. G. Adler im Literaturarchiv Marbach befindet, wird nach der bereits zitierten Verkündung der Regeln durch den Ältesten jäh mit der anonymen Erzählperspektive gebrochen ${ }^{22}$ :

21. - H.G. Adler, „Der Älteste“, in: Ders., Der Fürst des Segens, Bonn, Bibliotheca Christiana, 1964, hier S. 81.

22. - In der in Der Fürst des Segens enthaltenen Version von „Der Älteste“ fehlt dieser Abschnitt, der dem bereits zitierten Vortrag des Ältesten folgt. 
So etwa, nur noch viel länger ausgeführt und mit noch viel zahlreicheren Wiederholungen, als hier angedeutet, ging der Wortlaut des verlesenen Textes, von dem wir nur diese kurze Probe zu bieten wagen. Auch wären wir nicht in der Lage, aus den gewunden langen Sätzen, deren Vortrag mindestens eine Viertelstunde in Anspruch nahm, irgendeinen Sinn zu entnehmen, der nicht bereits in dem kurzen Stücke enthalten wäre, das wir gerade mitteilten. In immer wieder neuen Wiederholungen, in eintöniger Eindringlichkeit las und sang, rezitierte und deklamierte der Älteste gleichgültige (u n s gleichgültige!) Grußvorschriften.

Unvermittelt unterbricht hier der Erzähler die gewohnte Erzählperspektive aus der dritten Person und nimmt die Haltung der ersten Person Plural ein, womit er zugleich von der heterodiegetischen, also unbeteiligten, zur homodiegetischen Stimme wechselt. Somit offenbart er seine ambivalente Zugehörigkeit zu dem von ihm verachteten Kollektiv der passiven Zuhörer. Denn das plötzliche „uns“, ganz wie die unterbrochene Erzählperspektive des Wir in Eine Reise, kann kaum als Affirmation eines präsenten oder irgendwie positiv besetzten Kollektivs gewertet werden. Warum die Solidarität des Erzählers nicht schon früher seinen Leidensgenossen gilt, warum sie sich erst über den „Umweg' der beißenden Ironie zeigen kann - diese Fragen treffen den Kern einer ästhetischen Neuverhandlung des Wirs nach 1945.

\section{Literatur ohne Subjekt}

In seinem Essay „Streitgespräche, oder: Sätze bilden ,nach Auschwitz“" benennt der Philosoph Jean-François Lyotard implizit eine der Grundkonditionen der Heiterkeit nach 1945: Heiterkeit braucht Gemeinschaft, doch Gemeinschaft, selbst als denkerisches oder ästhetisches Konstrukt, ist, nach der Shoah, nicht mehr ungebrochen vorstellbar. „Streitgespräche“ handelt von der Möglichkeit eines (philosophischen) Diskurses, der im Bewusstsein der historischen Zäsur, die dieses Ereignis darstellt, an Auschwitz ,anschließt“23. Ohne Auschwitz ,die Zauberkraft des Negativen zuzuschreiben" 24 und abseits einer dialektischen Auflösung versucht Lyotard damit an Adornos Modell einer „negativen Dialektik“ und Jacques Derridas dekonstruktives Verfahren anzuknüpfen. „Das Modell ,Auschwitz' würde eine Erfahrung von Sprache bezeichnen, die dem spekulativen Diskurs Halt gebietet. Dieser könnte ,nach Auschwitz', das heißt, ,in Auschwitz' nicht fortgesetzt werden. Hier liegt ein Name vor, ,in' dem wir nicht oder nicht ganz

23. - J.-F. Lyotard, „Streitgespräche...“, a.a.O., S. 24.

24. - Ebd., S. 25. 
denken würden" 25 . Das Problem eines nicht zu bewältigenden negativen ,Rests', über den sich nicht sprechen lässt, ohne ihn zu affirmieren, stellt sich damit für Lyotard als das des „Name[ns] des Namenlosen“, und das nicht zuletzt, weil Auschwitz den Standpunkt, von dem aus normative Aussagen getroffen werden können, unterminiert. So fragt Lyotard: „,[h]at "Auschwitz" nicht deshalb keinen Namen, weil es der Eigenname einer Meta-Erfahrung ist, der der Unmöglichkeit des wir? Stimmt es nicht, daß es in den Lagern kein Subjekt im Plural gibt? Und daß, in Ermangelung eines derartigen Subjekts, "nach Auschwitz" kein Subjekt übrigbleibt, das geltend machen könnte, sich zu nennen, indem es diese "Erfahrung" benennt?"26? Weitergeführt könnte man sagen, dass es für Lyotard niemanden - vor allem kein Kollektiv - gibt, der oder das Auschwitz überlebt ${ }^{27}$. Die totale Atomisierung und Entmenschlichung der Einzelnen im Lager verbieten die Existenz eines Wirs, das sich anders als passiv gegenüber dem Befehl zu sterben gebärdet.

Ein Subjekt erübrigt sich vor allem dort, wo, wie in Eine Reise, der Befehl gilt „Sterbt, ich verfüge es" 28 („Du sollst nicht wohnen“" und „Das Leben ist verboten" 29 heißt es in Eine Reise). Dieser Befehl, so Lyotard, unterscheidet sich von anderen Fällen absoluter Autorität, weil der Empfänger des Befehls und dessen Sender in voneinander abgesonderten „Satzuniversen“ existieren. Wie die Lustigs zunächst verzweifelt versuchen, sich an die neue Ordnung anzupassen und sich zu spät bewusst werden, dass sie bereits verschwunden sind, bleibt dem Befehlsempfänger nach Lyotard nur die „Instanz des Referenten“:

Denn der Inhalt des Befehls ist in der Logik der Satzspiele so geartet, daß der Eigenname des Empfängers, wenn er gehorcht, nie wieder in den Empfänger- oder Senderinstanzen in den unmittelbar folgenden Sätzen wird erscheinen können. Er wird nur mehr in der Instanz des Referenten erscheinen können ${ }^{30}$.

Lyotard schlussfolgert, dass nur „die Identifizierung mit einem Wir [...], das fähig ist, als Sender- und Empfängerinstanz alle möglichen Befehle zu legitimieren, angefangen mit demjenigen, zu sterben“ 31 es dem Empfänger erlaubt, dem Todesurteil zu entgehen und ,gerade

25. - Ebd., S. 23.

26. - J.-F. Lyotard, „Streitgespräche...“, a.a.O., S. 35, 36.

27. - Diese Schlussfolgerung zieht Lyotard in einem Essay zu Hannah Arendt, siehe „Survivant“, in Jean-François Lyotard, Lectures d'enfance, Paris, Galilée, 1991.

28. - J.-F. Lyotard, „Streitgespräche...“, a.a.O., S. 35.

29. - H.G. Adler, Eine Reise, a.a.O., S. 34.

30. - J.-F. Lyotard, „Streitgespräche...“, a.a.O., S. 35.

31. - Ebd., S. 36. 
deshalb [zu] sterben“. „Er tauscht seinen einzigartigen Namen gegen ein kollektives Pronomen ein“ 32 .

Ganz ähnlich entfaltet sich in Adlers Texten eine „Literatur ohne Subjekt" gerade über eine mimetische Form der dunklen Ironie, die sich der tödlichen Logik von Befehl, Bürokratie und Zwangsgemeinschaft so vollständig angleicht, dass sie ihr allein über die Verabsolutierung und Vernichtung der Einzelnen im Kollektivpronomen wir ,entkommt“. Das Subjekt rettet sich und seine Fähigkeit zur Verbindung mit anderen, so könnte man sagen, indem es sich im Trojanischen Pferd eines an tödliche Logik angelehnten Wirs verbirgt. Aber gerade weil die totalitäre Struktur sich dem Erzähler aufzwingt, ihm diktiert, wie und was er ,in Ermangelung eines [pluralen] Subjekts“ der Erfahrung über die Lager und in ihrer Folge überhaupt noch schreiben kann, muss er ihr mit Heiterkeit begegnen ${ }^{33}$.

\section{Heiterkeit des Unmöglichen}

In einem Gedicht zu Ehren ihres Freundes H. G. Adler beschreibt Ilse Aichinger die eigentümliche Verbindung von wissenschaftli-

32. - Ebd.

33. - Damit ist Adler in der „Holocaust-Literatur“ freilich nicht allein. Harald Weinrich zitiert in seinem Essay zur Literaturgeschichte der Heiterkeit Lion Feuchtwangers Roman Die Geschwister Oppermann, in dem die Berliner Familie Oppermann noch angesichts Hitlers Machtübernahme 1933 ,[h]eiter, in Ruhe und Zuversicht" ihrem tödlichen Ende entgegenblickt. Das ironische Topos der jüdischen Familie, die in tiefster politischer Naivität ihre Hoffnung auf Staat und Vaterland setzt, die sich von keiner Ausschreitung und keiner Einschränkung ihres Lebens überzeugen lässt, die Koffer zu packen und noch bis zur letzten Minute fest daran glaubt, es handele sich um einen bloßen Irrtum, findet sich in einer Vielzahl von satirischen oder beinah satirischen jüdischen Romanen. Aharon Appelfeld lässt seine Kurgäste in Badenheim 1939 geduldig auf die von der „Sanitätsabteilung“ organisierten „Weiterreise“ in den Kurort Auschwitz warten. Der fünfzehnjährige György in Imre Kertész' Roman eines Schicksallosen, aus dessen nahezu fanatisch gutgläubiger Sicht den Lesern Deportation und Alltag in Auschwitz und Buchenwald präsentiert werden, ist um nichts naiver als seine Verwandten, die den Jungen und sich selbstzufrieden zum „unverbrüchlichen Vertrauen“" in die Barmherzigkeit Gottes und damit zur angeblich besonders jüdischen Tugend einer „opferwillige[n] Geduld“ aufrufen (Imre Kertész, Roman eines Schicksallosen, Berlin, Rowohlt, 1996, S. 26,7). In Adlers eigenem Roman Eine Reise wird die Familie Lustig wie viele andere rücksichtslos zusammengetrieben und völlig unvorbereitet in die Katastrophe gestürzt. Weil sich mit den „Fremdlingen“ an der Haustür nicht reden lässt - „Sagen Sie mal, kann man gar nichts machen? Ich möchte morgen noch was erledigen. Außerdem bin ich fest überzeugt, daß alles auf einem Irrtum beruht" - werden Vorbereitungen getroffen: Brote schmieren, Kuchen backen, Wohnung putzen. „Unvorhergesehenes Schicksal? Das kommt nicht in Betracht, ist auch nur Täuschung. Das Unbekannte findet nicht statt. Man hat sich genau an die Vorschriften zu halten“ (Eine Reise, a.a.O., S. 12, 13). Siehe auch Harald Weinrich, Kleine Literaturgeschichte der Heiterkeit, München, C.H. Beck, 2001, hier S. 43. 
cher Präzision und empfindsamer Poetik, die seine „lyrische Ironie“34 ausmacht. Sie bemerkt in „Versuch zu danken“:

\author{
Die genaue Ahnung, \\ das genaue Wissen, \\ Schutz und Zuflucht. \\ Die Helligkeit beim Eintritt \\ macht gewiß: \\ Hier ist einer durch die Schwärze gegangen \\ und bleibt ${ }^{35}$.
}

Es ist die „Helligkeit beim Eintritt“ in das fiktionale Gebäude, die uns wissen lässt, „Hier ist einer durch die Schwärze gegangen“. Aichinger spricht von einem „Versuch, zu danken“ sowohl für die Genauigkeit des Wissens, die sie Adler zu verdanken hat, und der Ahnung, in der das lyrische Ich „Schutz und Zuflucht" findet, als auch für sein Verweilen in der „Schwärze“ (,und bleibt“). Paul Celans Rede von den „tausend Finsternisse[n]"36, durch welche die Sprache „hindurchgehen“ muss, klingt hier an und doch ist der Eindruck der Helligkeit wohl nur für Adlers Texte charakteristisch.

Helligkeit ist ein Wort, das immer wieder in Reaktionen auf dieses Werk auftaucht. Ingeborg Drewitz zum Beispiel beobachtet in einer verblüffend ähnlichen Formulierung die Scham derer, die sich wundern, „daß einer nach solch einem Leben so schreiben kann, der Helligkeit zugewandt" 37 . Auch die ersten Rezensenten wie Elias Canetti und Heimito von Doderer schwärmen von Leichtigkeit, Transzendenz, ja von der Heiterkeit dieser Literatur. Es handele sich bei Adler, so lobt von Doderer 1964, um einen von jenen Überlebenden, die uns nichts nachtragen.

Eine Ballade klagt nicht an, sie befreit den Autor, sie befreit den Leser, sie macht den Gegenstand gewichtslos, sei er wie er sei (und hier grenzt die Sache ans Unglaubliche), gewichtslos und schwebend, ohne $\mathrm{ihm}$ von seiner Gewichtigkeit das Geringste zu nehmen. Ein ganzer Berg von Schrecken wird zum Liede ${ }^{38}$.

34. - So definiert Adler selbst seine Methode in einem Brief an einen Rezensenten seines Romans Eine Reise: Brief an Theodor Sapper vom 21.1.1974, Literaturarchiv Marbach.

35. - Ilse Aichinger, „Versuch zu danken“, in: Andreas W. Mytze (Hrsg.), H.G. Adler zum 75. Geburtstag, Europäische Ideen, 60 (1985), S. 1.

36. - Paul Celan, ,,Ansprache anlässlich der Entgegennahme des Literaturpreises der Freien Hansestadt Bremen“, in: Paul Celan, Gesammelte Werke 3, Frankfurt a.M., Suhrkamp, 1983, S. 185.

37. - Ingeborg Drewitz in: Andreas W. Mytze (Hrsg.), H.G. Adler zum 75. Geburtstag, a.a.O. S. 1.

38. - Heimito von Doderer, „Die Schule des Lesers. Notizen zu H. G. Adlers 
Solche Lobeshymnen sind im Kontext der Zeit natürlich zutiefst problematisch und können als Abwehr gegen den „Skandal“ der Opfer gedeutet werden. Der Literaturkritiker und frühe Fürsprecher Adlers Jürgen Serke formuliert diese Erkenntnis bereits 1987 deutlicher als die neuere Adler-Forschung: „Das Wiederauftauchen des Opfers“ bedeutet laut Serke „,nicht nur de[n] Skandal für die sich normalisierenden Verfolger“, sondern auch „für alle anderen“. Zugrunde liege hier eine Angst vor der Kontamination mit dem Tod: „Die Welt hat Angst vor jenem, der das Ende des Lebens wieder in das Leben trägt. Die Welt möchte davon nicht behelligt werden. Er ist für sie infiziert mit dem Tod"39. Adler erlebte diese Angst am eigenen Leib; seine Bemühungen, ein Publikum und Verlage zu finden, blieben weitgehend erfolglos ${ }^{40}$.

Die Tatsache, dass Adler zu Lebzeiten relativ unbekannt, seine Romane ungelesen blieben, scheint in eklatantem Widerspruch mit der kritischen Wahrnehmung seiner versöhnenden Botschaft zu stehen. Auf der einen Seite steht hier die versuchte Vereinnahmung seiner Person durch die Versöhnungsideologie der Nachkriegszeit, die danach trachtete, den Juden Adler, der in wissenschaftlichen Artikeln unverblümt über Auschwitz und Langenstein berichtet hatte, zum Teil des ,externalisierten Gewissens' der Bundesrepublik zu stilisieren. Der damalige Bundespräsident Theodor Heuss etwa (ein Unterzeichner des Ermächtigungsgesetzes und einstmaliger Antisemit) lud Adler nach dem internationalen Erfolg seiner Theresienstadt-Studie zu sich ein ${ }^{41}$, um ihn bei dieser Gelegenheit zu bitten, ihm „Literaturangaben über die Anzahl der umgekommenen Juden“42 zukommen zu lassen. Wie viele andere jüdische Nachkriegsschriftsteller, die sich nicht mit aller Kraft gegen die Vereinnahmung wehrten, wurde Adlers literarisches

Erzählung 'Eine Reise' (Verlag Bibliotheca Christiana, Bonn)“, Forum 6:121 (Januar 1964), S. 37. Auch für Elias Canetti hat Adlers Roman eine klärende Wirkung: ,,Eine Reise' [...] halte ich für ein Meisterwerk. Es ist in besonders schöner, reiner Prosa geschrieben, jenseits von Groll und Bitterkeit, Ausdruck einer essentiellen Läuterung“. Zitiert in Franz Hocheneder, „Aufzeichnungen einer Displaced Person. Werk und Nachlass von H.G. Adler (1910-1988)“, Literatur und Kritik, Nov. 1998, S. 52.

39. - J. Serke, Böhmische Dörfer, a.a.O., S. 328.

40. - Seine Romane Panorama und Eine Reise mussten jeweils elf und zwanzig Jahre auf ihre Veröffentlichung in kleinen Verlagen warten. Viele andere literarische Arbeiten bleiben bis heute unveröffentlicht. Für Serke bedeutet die Ablehnung von Adlers Werk einen beispielslosen „Skandal, für den die deutschen Verlage verantwortlich zeichnen“. Noch 1987 lehnt der Suhrkamp Verlag Eine Reise als „,nicht eigentlich gelungen" ab. Ebd., S. 327.

41. - Siehe Marcel Atze, ,,Was hast du ihm gesagt, dem Mörder?` H. G. Adler“, in: Irmtraud Wojak (Hrsg.), Auschwitz-Prozeß 4Ks2/63, Fritz Bauer Institut, Frankfurt a. M., Snoeck, 2004, S. 649.

42. - Brief von H. G. Adler an Theodor W. Adorno vom 29.10.1956, Literaturarchiv Marbach. 
Schaffen in der Bundesrepublik und in Österreich fast ausschließlich im Dienste der Versöhnung wahrgenommen. In der Sammlung Vorletzte Worte. Schriftsteller schreiben ihren eigenen Nachruf (1970) etwa erscheint Adlers fiktionaler Nachruf neben denen humoristisch-postmoderner Autoren wie Peter Chotjewitz und Frank Arnau sowie ehemaliger NS-Parteigänger wie Karl Krolow und Jürgen Thorwald und den Veteranen der NS-Unterhaltungsbranche Hans Leip und Alexander Lernet-Holenia. H. G. Adler, der seine gesamte Familie in den Lagern verlor, schreibt hier in der dritten Person weniger über seinen Tod als über die Schwierigkeit, überhaupt Abnehmer für seine literarischen Texte zu finden. Andererseits passte Adlers literarisches Schaffen also offensichtlich - trotz „Helligkeit“ - nicht in die staatlich und kirchlich geförderte Vergebungskultur. So urteilt Ingeborg Bachmann in einem Brief an Adlers Frau Bettina vom Oktober 1951: „mit diesem Roman [Die Ansiedlung ${ }^{43}$ ], den ich persönlich sehr schätze, lässt sich, gerade in Österreich, wohl schwer etwas anfangen"44.

Paradoxerweise ist es womöglich gerade die ,Heiterkeit' der Adlerschen Texte - jene „Helligkeit beim Eintritt“ -, die sich, in Verbindung mit der bereits diskutierten Infragestellung von Gemeinschaftlichkeit, der ideologischen Verwertung und dem Verständnis vieler Leser in der Nachkriegszeit entzieht. Genau das lässt zumindest die Kritik eines weiteren Adler-Lesers vermuten, dessen Beurteilung sich an einer entscheidenden Stelle mit der gängigen Interpretation trifft - und das trotz diametral entgegengesetzter politischer Überzeugungen. In Adler, so urteilt Theodor Adorno in „Meditationen zur Metaphysik“, finde sich „Einer“, dem die Heiterkeit zur Zwangshandlung geworden ist:

Einer, der mit einer Kraft, die zu bewundern ist, Auschwitz und andere Lager überstand, meinte mit heftigem Affekt gegen Beckett: wäre dieser in Auschwitz gewesen, er würde anders schreiben, nämlich, mit der Schützengrabenreligion des Entronnenen, positiver. Der Entronnene hat anders recht, als er es meint; Beckett, und wer sonst noch seiner mächtig blieb, wäre dort gebrochen worden und vermutlich gezwungen, jene Schützengrabenreligion zu bekennen, die der

43. - Der unvollendete Roman „Die Ansiedlung“ handelt wiederum genau von Adlers Thema, der unmöglichen und unhaltbaren Gemeinschaft. In einer stark von Kafka inspirierten Weise erzählt der Roman von der Dystopie eines Erziehungsheimes für Jugendliche, das von einer „Behörde der Ansiedlung“ zu Tode verwaltet wird: „Gewiß geht es bei uns nicht paradiesisch zu, gewisse Mängel sind unvermeidlich, aber wir stellen uns stolz und bescheiden jedem Vergleich. Grund genug, um dafür dankbar zu sein, daß die Ansiedlung besteht, wir brauchen uns ihrer nicht zu schämen“. H. G. Adler, „Die Ansiedlung“, Literaturarchiv Marbach, AII 49, S. 1.

44. - Brief von Ingeborg Bachmann an Bettina Adler vom 27.10.1951, Literaturarchiv Marbach. 
Entronnene in die Worte kleidete, er wolle den Menschen Mut geben: als ob das bei irgendeinem geistigen Gebilde läge ${ }^{45}$.

Helligkeit und Heiterkeit werden Adorno zur „Schützengrabenreligion“ und damit zu einer Angelegenheit kollektiver Absolution. Dass Adler „Mutgeben“ gerade als Mut, die inneren Brüchigkeiten von Gemeinschaft während und nach der Shoah auszusprechen verstehen könnte, kommt Adorno nicht in den Sinn. Sein bitterer Vorwurf, demzufolge Adler seine Erfahrung in den Lagern metaphysisch auszubeuten sucht, stimmt mit seiner These in „Ist die Kunst heiter?“ überein, wo er Heiterkeit zunächst mit der idealistischen Wirkungsästhetik in Verbindung bringt. Beide, Heiterkeits- und Wirkungsästhetik, orientieren sich an den äußerlichen Effekten der Kunst auf den Betrachter. Adorno kontert, die Kunst solle, ohne sich um ihren vermeintlichen Zweck oder ihren Einfluss zu kümmern, ihre Opposition gegen die Zweckmäßigkeit so drastisch verkörpern, wie es ihr eigen ist. Mut zu stiften gehört damit nicht zum eigentlichen Wesen der Kunst, sondern ordnet sie erneut unter das Gesetz der „Selbsterhaltung“: „Das Ohne Zweck der Kunst ist ihr Entronnensein aus den Zwängen von Selbsterhaltung“46. (Wenig überraschend ist Adornos erstes Beispiel in seinem Essay zur Frage der Heiterkeit wiederum Beckett.) Adornos berühmte Revidierung seines Satzes von der Dichtung nach Auschwitz in „Ist die Kunst heiter?“, wo er einschränkt, die Kunst nach Auschwitz sei, wenn schon nicht unmöglich, so wenigstens unmöglich heiter, scheint seine Andeutungen kontra Adler zu bestätigen. Denn eine Kunst, die sich den Gestus der „Güte menschlichen Verstehens" zu eigen macht, könne nur in "Zynismus“ ausarten - der gleiche Zynismus, so könnte man weiterdenken, der den „Entronnenen“ dazu verleitet, zu spekulieren, wie es Beckett in Auschwitz ergangen wäre.

Und doch lässt sich mit Adorno auch gegen Adorno argumentieren: Die gemeinschaftskritische Ironie der Adlerschen Texte, von den ,seltsamen Namen“ bis zur Reise der Lustigs, kann im Sinne des in „Ist die Kunst heiter?“ prognostizierten „Absterben[s] der Alternative von Heiterkeit und Ernst" "47 als Heiterkeit des Unmöglichen gelesen werden. Heiterkeit überlebt, spekuliert Adorno am Ende seines Essays, als „Selbstkritik [der Kunst], als Komik der Komik“. Anders als in der klassischen Ästhetik ist die Kunst demnach nicht mehr der Gemeinschaft

45. - Theodor W. Adorno, Negative Dialektik, Frankfurt a.M., Suhrkamp, 1997, S. 360,1

46. - Ebd., S. 600.

47. - T. W. Adorno, „Ist die Kunst heiter?“, in: Ders., Noten zur Literatur, Frankfurt a.M., Suhrkamp, 1974, S. 605. 
verpflichtet, sei es als Entertainment oder als ,Wille zur Gemeinschaft“48, und sie verweigert ihre Rolle als Identifizierungsstifter.

Während sich Heiterkeit als Eigenschaft der Kunst und Ausdruck eines Kollektivs - wie er sich etwa im Gelächter, im Witz und der Gesellschaftssatire artikuliert - in der Nachkriegszeit in peinlicher Nähe zu jenem Gemeinschaftswillen findet, der mit der Errichtung des sogenannten „Dritten Reichs“ noch das Lachen in den Dienst des Staates stellte ${ }^{49}$, kann Adlers Helligkeit das Verhältnis von Kunst und Intersubjektivität neu beleuchten. Wo sich aber Heiterkeit als Selbst- und Gemeinschaftskritik neu erfindet, wie es in zahlreichen literarischen Projekten jüdischer Nachkriegsautoren von Imre Kertész und Edgar Hilsenrath bis Aharon Appelfeld und Adler deutlich wird, dissoziiert sie sich von ihrer immanenten Funktion. Heiterkeit erscheint dann als Bemühen, den Status des Ästhetischen wiederherzustellen und seine Verbindung zu gesellschaftlicher Relationalität zu überdenken.

\section{Zum Abschied winken}

Am Ende seines Wegs von Ruhenthal über Leitenberg und Unkenburg, durch die ehemals vertrauten Orte seiner Kindheit, und nach einer Reihe von Begegnungen mit unwilligen Zeugen, Verwaltern der neuen Ordnung und neuen Freunden, ist nur mehr Paul, der Sohn der Familie Lustig, übrig. Die Reise ist zu Ende, ein Zug soll ihn wegbringen, zurück zum Anfang seiner Verfolgung. Er scheint allein zu sein, ist es aber nicht ganz:

48. - „Nützen wollen die Dichter, die Dichter wollen ergötzen“, so der römische Dichter Horaz, zitiert von Emil Staiger, dessen Verteidigung einer ,geselligen“ Heiterkeit in der Kunst in seiner berüchtigten Rede zur Verleihung des Literaturpreises der Stadt Zürich im Jahr 1966 auch deswegen auf Ablehnung unter linken Schriftstellern wie Max Frisch und Peter Handke stieß, weil der bekannte Schweizer Germanist das Verhältnis von Gemeinschaft und Kunst als so vollkommen unproblematisch präsentierte, als seien die letzten dreißig Jahre spurlos an ihm vorübergezogen. Die littérature engagée - ,eine Entartung jenes Willens zur Gemeinschaft, der Dichter vergangener Tage beseelte“ verstoße gegen die Aufgabe der Kunst, stets ,im Namen der Menschengemeinschaft“ zu sprechen, und der des Künstlers, in dessen „Urteil [...] der Wille zu einer möglichen, auf den Fundamenten der Sittlichkeit gegründeten Menschengesellschaft fühlbar“ sein müsse. Emil Staiger, „Literatur und Öffentlichkeit“, Neue Zürcher Zeitung, 20. Dezember 1966, https://static.nzz.ch/download/pdf/Emil_Staiger_Rede.pdf.

49. - Patrick Merziger z.B. argumentiert, dass Satire im NS-Staat nahezu gänzlich verschwand, weil sie sich als unverträglich mit dem harmonischen Bild der Volksgemeinschaft erwies. Vgl. „Humour in the Volksgemeinschaft: The Disappearance of Destructive Satire in National Socialist Germany“, in: Martina Kessel und Patrick Merziger (Hrsg.), The Politics of Humour: Laughter, Inclusion and Exclusion in the Twentieth Century, Toronto, University of Toronto Press, 2012, S. 131-152. 
Die rote Kappe hat es versprochen. Paul soll sich in die Reihe der Wartenden stellen. Der Schwarm läßt ihn voran. Paul dankt, er schiebt den Koffer vor, die freie Hand umklammert schon die kühle Stange. Vielleicht verstehen es die Menschen, warum er es besonders eilig hat. Er glaubt, sie winken ihm zu einer guten Reise, weil der Abfall überwunden ist ${ }^{50}$.

Dass die Anderen ,vielleicht verstehen“, dass sie winken, darin liegen H. G. Adlers Mut und der Grund seiner Heiterkeit. 